PROCEEDINGS OF THE

AMERICAN MATHEMATICAL SOCIETY

Volume 134, Number 4, Pages 983-987

S 0002-9939(05)08246-8

Article electronically published on September 28, 2005

\title{
ON THE PRODUCTS OF NASH SUBVARIETIES BY SPHERES
}

\author{
ALESSANDRO TANCREDI AND ALBERTO TOGNOLI
}

(Communicated by Michael Stillman)

\begin{abstract}
We show that the product of any sphere by any compact connected component of a real algebraic variety is Nash isomorphic to a real algebraic variety, and we deduce such a result for some non-compact components, too. It follows also that the product of any sphere by any compact global Nash subvariety of $\mathbb{R}^{n}$ is Nash isomorphic to a real algebraic variety.
\end{abstract}

\section{INTRODUCTION AND PRELIMINARY REMARKS}

We recall some results and definitions that we shall use in the following.

By a real algebraic variety we mean a closed real algebraic subvariety of some $\mathbb{R}^{n}$, i.e. the zero set in $\mathbb{R}^{n}$ of polynomials of $\mathbb{R}\left[x_{1}, \ldots, x_{n}\right]$, which is a locally ringed space, endowed with its Zariski topology and its sheaf of regular functions (see [BCR] $)$. Unless explicitly stated otherwise we consider the Euclidean topology on algebraic varieties. We remark that every Zariski open subset of a real algebraic variety is biregularly isomorphic to a variety.

Let $\Omega$ be an open subset of $\mathbb{R}^{n}$; a Nash function on $\Omega$ is a real analytic function which verifies, on $\Omega$, an algebraic relation over the polynomial ring $\mathbb{R}\left[x_{1}, \ldots, x_{n}\right]$ (see e.g. $[\mathrm{BCR}]$ ).

A Nash subvariety $X$ of $\mathbb{R}^{n}$ is a locally closed subset which is, locally, the zero set of a finite number of Nash functions. Nash functions on open sets of $X$ are local restrictions of Nash functions on open sets of $\mathbb{R}^{n}$.

A global Nash subvariety $X$ of $\mathbb{R}^{n}$ is a closed Nash subvariety which is the zero set of Nash functions defined on an open semi-algebraic neighborhood of $X$. Of course every connected component of a real algebraic variety is a global Nash subvariety.

A Nash map between Nash subvarieties is locally defined by Nash functions. A Nash isomorphism of Nash subvarieties is a bijective Nash map whose inverse is also a Nash map. By a global Nash isomorphism we mean an isomorphism which extends, together with its inverse, to Nash maps on open neighborhoods of the subvarieties. We remark that closed Nash subvarieties and Nash isomorphisms between them can fail to be global (see e.g. [TT]).

In this paper we prove, by simple geometric arguments, that for any sphere $S^{r}$ and any compact connected component $X^{\prime}$ of a real algebraic variety $X$, there exists

Received by the editors February 9, 2004 and, in revised form, November 4, 2004.

2000 Mathematics Subject Classification. Primary 14P05; Secondary 14P20, 58A07.

Key words and phrases. Nash function, global Nash subvariety.

The authors are members of GNSAGA of INDAM. This work was partially supported by MIUR and by European Contract HPRN-CT-2001-00271.

(C)2005 American Mathematical Society Reverts to public domain 28 years from publication 
a global Nash isomorphism $\nu$ of $S^{r} \times X^{\prime}$ onto a real algebraic variety. Moreover, if $X_{1}$ is an algebraic subvariety of $X$, such an isomorphism maps $S^{r} \times\left(X^{\prime} \cap X_{1}\right)$ onto an algebraic subvariety of $\nu\left(S^{r} \times X^{\prime}\right)$. It follows a sufficient condition in order that the product of a sphere by a non-compact component of a real algebraic variety be globally Nash isomorphic to a real algebraic variety: in particular, this is the case of a connected component whose complement is compact. Finally, by using the Artin-Mazur Theorem, we prove that the product of any sphere by any compact global Nash subvariety $X$ is globally Nash isomorphic to a real algebraic variety. The question if $X$ itself is isomorphic to an algebraic variety is a deeper open question. The answer is affirmative when $X$ is smooth ([To1, [To2]) or it has isolated singularities $([\mathrm{AK}]$ ), but the proofs need different and stronger methods.

\section{RESUlts AND PROOFS}

Lemma 2.1. Let $Y$ be a real algebraic variety in $\mathbb{R}^{m}$ and let $Y_{1}$ be an algebraic subvariety of $Y$. Let $Y^{\prime}$ be a compact connected component of $Y$ such that $Y^{\prime} \subset$ $\left\{x \in \mathbb{R}^{m} \mid\|x\|<1\right\}$ and $Y-Y^{\prime} \subset\left\{x \in \mathbb{R}^{m} \mid\|x\|>3\right\}$ and let $Y_{1}^{\prime}=Y^{\prime} \cap Y_{1}$.

Then there exist an algebraic variety $X$ in $\mathbb{R}^{m}$ which has only two connected components, $X^{\prime}$ and $X^{\prime \prime}$, and an algebraic subvariety $X_{1}$ of $X$ which is the union of two disjoint Nash subvarieties, $X_{1}^{\prime}$ and $X_{1}^{\prime \prime}$, such that:

i) $X_{1}^{\prime} \subset X^{\prime}, X_{1}^{\prime \prime} \subset X^{\prime \prime}$;

ii) $X^{\prime}$ and $X_{1}^{\prime}$ are symmetric with respect to the origin of $\mathbb{R}^{m}$ to $X^{\prime \prime}$ and $X_{1}^{\prime \prime}$, respectively;

iii) $X^{\prime} \subset\left\{x \in \mathbb{R}^{m} \mid\|x\|<1, x_{m}>0\right\}, X^{\prime \prime} \subset\left\{x \in \mathbb{R}^{m} \mid\|x\|<1, x_{m}<0\right\}$;

iv) there exists a global Nash isomorphism from $Y^{\prime}$ onto $X^{\prime}$ which maps $Y_{1}^{\prime}$ onto $X_{1}^{\prime}$.

Proof. We can assume $Y^{\prime} \subset\left\{x \in \mathbb{R}^{m} \mid\|x\|<1, x_{m}>0\right\}, Y-Y^{\prime} \subset\{x \in$ $\left.\mathbb{R}^{m} \mid\|x\|>1\right\}$ and $\mathbb{R}^{m}$ is canonically embedded into $\mathbb{R}^{m+1}$. Let $S$ be the sphere of radius 1 and center the origin $O$ of $\mathbb{R}^{m+1}$ and let us consider the cylinders $C(Y), C\left(Y^{\prime}\right), C\left(Y_{1}\right), C\left(Y_{1}^{\prime}\right)$ over $Y, Y^{\prime}, Y_{1}, Y_{1}^{\prime}$, respectively. Let $Z=S \cap C\left(Y^{\prime}\right)$, $Z_{1}=S \cap C\left(Y_{1}^{\prime}\right)$. Since $S \cap C(Y)=S \cap C\left(Y^{\prime}\right)$ and $S \cap C\left(Y_{1}\right)=S \cap C\left(Y_{1}^{\prime}\right), Z$ and $Z_{1}$ are algebraic varieties: $Z$ has only two connected components, $Z^{\prime}=\left\{x \in Z \mid x_{m+1}>0\right\}$ and $Z^{\prime \prime}=\left\{x \in Z \mid x_{m+1}<0\right\} ; Z_{1}$ is the union of the two Nash subvarieties $Z_{1}^{\prime}=Z^{\prime} \cap Z_{1}$ and $Z_{1}^{\prime \prime}=Z^{\prime \prime} \cap Z_{1}$. Clearly the canonical projection $\mathbb{R}^{m+1} \longrightarrow \mathbb{R}^{m}$ induces a global Nash isomorphism $Z^{\prime} \longrightarrow Y^{\prime}$ which maps $Z_{1}^{\prime}$ onto $Y_{1}^{\prime}$.

Let $C(O, Z)$ (resp. $\left.C\left(O, Z_{1}\right)\right)$ be the union of the lines joining $O$ to points of $Z$ (resp. $\left.Z_{1}\right)$; every line $l$ of $C(O, Z)$ (resp. $\left.C\left(O, Z_{1}\right)\right)$ meets $Z$ (resp. $\left.Z_{1}\right)$ at only one point.

Let $\tilde{Z}, \tilde{S}$ be the Zariski closures of $Z$ and $S$ in $\mathbb{C}^{m+1}$ and let $\hat{\tilde{Z}}$, $\hat{\tilde{S}}$ be their projective closures in $\mathbb{P}^{m+1}(\mathbb{C})$. Let $C(O, \hat{\tilde{Z}})$ be the union of the lines joining $O$ to points of $\hat{\tilde{Z}}$ in $\mathbb{P}^{m+1}(\mathbb{C}): C(O, \hat{\tilde{Z}})$ is a projective complex algebraic variety. Let us consider the canonical embeddings $\mathbb{R}^{m+1} \subset \mathbb{C}^{m+1} \subset \mathbb{P}^{m+1}(\mathbb{C})$. Since every real line of $C(O, \hat{\tilde{Z}})$ meets $\hat{\tilde{Z}}$ at points of $Z$, we have $C(O, \hat{\tilde{Z}}) \cap \mathbb{R}^{m+1}=C(O, Z)$, so that $C(O, Z)$ is an algebraic variety in $\mathbb{R}^{m+1}$. In the same way we can conclude that $C\left(O, Z_{1}\right)$ is an algebraic subvariety of $C(O, Z)$.

The set $X=\left\{x \in C(O, Z) \mid x_{m+1}=1\right\}$ is an algebraic variety with only two connected components, $X^{\prime}=\left\{x \in X \mid x_{m}>0\right\}$ and $X^{\prime \prime}=\left\{x \in X \mid x_{m}<0\right\}$, while the set $X_{1}=\left\{x \in C\left(O, Z_{1}\right) \mid x_{m+1}=1\right\}$ is an algebraic variety which is the union of 
the two Nash subvarieties $X_{1}^{\prime}=X^{\prime} \cap C\left(O, Z_{1}\right)$ and $X_{1}^{\prime \prime}=X^{\prime \prime} \cap C\left(O, Z_{1}\right)$. Clearly the projection from $O$ induces a Nash isomorphism from an open neighborhood of $Z^{\prime}$ in $S$ onto an open neighborhood of $X^{\prime}$ in the hyperplane $x_{m+1}=1$. It follows that there exists a global Nash isomorphism from $Z^{\prime}$ onto $X^{\prime}$ which maps $Z_{1}^{\prime}$ onto $X_{1}^{\prime}$. Moreover $X^{\prime}$ and $X_{1}^{\prime}$ are symmetric, with respect to the point $(0, \ldots, 0,1)$, to $X^{\prime \prime}$ and $X_{1}^{\prime \prime}$, respectively. By taking the projections onto $\mathbb{R}^{m}$ we get the conclusion.

Remark 2.2. Let $Y$ be an algebraic variety and let $D$ be an open disk of $\mathbb{R}^{n}$. It may be of some interest to remark that the topological space obtained by gluing two copies of $Y \cap \bar{D}$ along the boundary of $D$ is homeomorphic to an algebraic variety. Indeed, as in the previous lemma, it is homeomorphic to $C(Y) \cap S$, where $S$ is the boundary of the disk $B$ of $\mathbb{R}^{n+1}$ such that $D=B \cap \mathbb{R}^{n}$.

Theorem 2.3. Let $X$ be a real algebraic variety and let $X_{1}$ be an algebraic subvariety of $X$. Let $X^{\prime}$ be a compact connected component of $X$ and let $X_{1}^{\prime}=X^{\prime} \cap X_{1}$. Then, for any sphere $S^{r}$, there exists a Nash map $\nu: S^{r} \times X^{\prime} \longrightarrow \mathbb{R}^{N}$ such that $\nu\left(S^{r} \times X^{\prime}\right)$ and $\nu\left(S^{r} \times X_{1}^{\prime}\right)$ are algebraic subvarieties of $\mathbb{R}^{N}$ and $\nu: S^{r} \times X^{\prime} \longrightarrow$ $\nu\left(S^{r} \times X^{\prime}\right)$ is a global Nash isomorphism.

Proof. We assume that $X$ is an algebraic subvariety of $\mathbb{R}^{m}$ and that $\mathbb{R}^{m}$ is canonically embedded into $\mathbb{R}^{m+1}$. We will reduce to the hypothesis of Lemma 2.1 by following the method used in Wa. We can find an open disk $B$ of $\mathbb{R}^{m+1}$ with center the origin and radius $b$ such that $X^{\prime} \subset B$. Let $c$ be the distance from $X^{\prime}$ to the boundary of $B$ and let $a>3(b+c)$ be a real number; let $D$ be the open disk of $\mathbb{R}^{m+1}$ with center the origin and radius $a$. There exists a continuous function $f: \bar{D} \cap \mathbb{R}^{m} \longrightarrow \mathbb{R}$ such that $f(x)=0$ for $x \in X^{\prime}$ and $f(x)=a+c$ for $x \in\left(X-X^{\prime}\right) \cap \bar{D}$. By the Weierstrass approximation theorem, there exists a polynomial $F \in \mathbb{R}\left[x_{1}, \ldots, x_{m}\right]$ such that $\|f-F\|_{\bar{D} \cap \mathbb{R}^{m}}<c$. Let $Y$ and $Y_{1}$ be the graph of the regular functions $\left.F\right|_{X}$ and $\left.F\right|_{X_{1}}$, and let $Y^{\prime}$ and $Y_{1}^{\prime}$ be the graph of the Nash functions $\left.F\right|_{X^{\prime}}$ and $\left.F\right|_{X_{1}^{\prime}}$. Clearly $Y^{\prime}$ is a Nash subvariety which is a compact connected component of the algebraic variety $Y$, and the canonical projection $\mathbb{R}^{m+1} \longrightarrow \mathbb{R}^{m}$ induces global Nash isomorphisms $Y^{\prime} \longrightarrow X^{\prime}$ and $Y_{1}^{\prime} \longrightarrow X_{1}^{\prime}$. Moreover, by construction, $Y^{\prime}$ is contained in an open disk of $\mathbb{R}^{m+1}$ with center the origin and radius $b+c$ and $Y-Y^{\prime}$ is contained in $\mathbb{R}^{m+1}-\bar{D}$.

By Lemma 2.1 it follows that we can reduce the proof to the case where $X$ is contained in $\left\{x \in \mathbb{R}^{m} \mid\|x\|<1\right\}$, has only two connected components, $X^{\prime}=$ $\left\{x \in X \mid x_{m}>0\right\}, X^{\prime \prime}=\left\{x \in X \mid x_{m}<0\right\}$, and that $X_{1}$ is the union of two Nash subvarieties $X_{1}^{\prime}$ and $X_{1}^{\prime \prime}$, with $X_{1}^{\prime} \subset X^{\prime}$ and $X_{1}^{\prime \prime} \subset X^{\prime \prime}$. Moreover $X^{\prime}$ and $X_{1}^{\prime}$ are symmetric with respect to the origin of $\mathbb{R}^{m}$ to $X^{\prime \prime}$ and $X_{1}^{\prime \prime}$, respectively.

Let us consider the algebraic subvarieties of $\mathbb{R}^{n} Z=S^{r} \times X$ and $Z_{1}=S^{r} \times X_{1}$, where $n=m+r+1$. $Z$ has only two connected components, $Z^{\prime}=S^{r} \times X^{\prime} \subset\{x \in$ $\left.\mathbb{R}^{n} \mid x_{n}>0\right\}$ and $Z^{\prime \prime}=S^{r} \times X^{\prime \prime} \subset\left\{x \in \mathbb{R}^{n} \mid x_{n}<0\right\}$, which are symmetric with respect to the origin $O$ of $\mathbb{R}^{n}$. Moreover, $Z_{1}$ is an algebraic subvariety of $Z$ which is the union of the two Nash subvarieties $Z_{1}^{\prime}=S^{r} \times X_{1}^{\prime} \subset Z^{\prime}$ and $Z_{1}^{\prime \prime}=S^{r} \times X_{1}^{\prime \prime} \subset Z^{\prime \prime}$. Furthermore $Z_{1}^{\prime}$ and $Z_{1}^{\prime \prime}$ are symmetric with respect to the origin of $\mathbb{R}^{n}$.

Let us consider the regular map $\nu: \mathbb{R}^{n} \longrightarrow \mathbb{R}^{N}$ defined by

$$
\nu:\left(x_{1}, \ldots, x_{n}\right) \longmapsto\left(\ldots, x_{i} x_{j}, \ldots\right)_{i \leq j},
$$


where $N=\left(\begin{array}{c}n+1 \\ 2\end{array}\right)$. The map $\nu$ is injective of rank $n$ on the half space $\left\{x \in \mathbb{R}^{n} \mid x_{n}>\right.$ $0\}$. It follows that $\nu\left(Z^{\prime}\right)=\nu(Z)$ and $\nu\left(Z_{1}^{\prime}\right)=\nu\left(Z_{1}\right)$ are Nash subvarieties of $\mathbb{R}^{N}$ globally Nash isomorphic to $Z^{\prime}$ and $Z_{1}^{\prime}$, respectively.

Let $\tilde{Z}$ (resp. $\tilde{Z}_{1}$ ) be the Zariski closure of $Z$ (resp. $\left.Z_{1}\right)$ in $\mathbb{C}^{n}$ and let us consider the extension $\tilde{\nu}$ of $\nu$ to $\mathbb{C}^{n}$. Since $\tilde{\nu}$ is regular and proper, $\tilde{\nu}(Z)$ and $\tilde{\nu}\left(Z_{1}\right)$ are closed constructible sets and as a result they are algebraic subvarieties of $\mathbb{C}^{N}$ (see e.g. [Lo] $)$.

Moreover, for any $z \in \mathbb{C}^{n}, \tilde{\nu}(z) \in \mathbb{R}^{N}$ if and only if all the components of $z$ are real numbers or complex numbers whose real parts are zero. Since such complex points cannot belong to complex spheres, it follows that $\nu(Z)=\tilde{\nu}(\tilde{Z}) \cap \mathbb{R}^{N}$ and $\nu\left(Z_{1}\right)=\tilde{\nu}\left(\tilde{Z}_{1}\right) \cap \mathbb{R}^{N}$, and so the conclusion follows.

Corollary 2.4. Let $T$ be a connected subset of $\mathbb{R}^{n}$ such that its closure $\bar{T}$ is a compact connected component of an algebraic variety $X$ in $\mathbb{R}^{n}$ and $\bar{T}-T$ is the intersection of $\bar{T}$ with an algebraic subvariety $X_{1}$ of $X$. Then, for any sphere $S^{r}$, the product $S^{r} \times T$ is globally Nash isomorphic to an algebraic variety.

Proof. Let $X^{\prime}=\bar{T}$ and $X_{1}^{\prime}=\bar{T}-T$. By Theorem 2.3 there exists a real algebraic variety $Y$ and a global Nash isomorphism $\nu: S^{r} \times X^{\prime} \longrightarrow Y$ such that $\nu\left(S^{r} \times X_{1}^{\prime}\right)$ is an algebraic subvariety of $Y$. Since there exists a biregular isomorphism from $Y-\nu\left(S^{r} \times X_{1}^{\prime}\right)$ onto an algebraic variety, the conclusion follows.

Corollary 2.5. Let $T$ be a connected component of a real algebraic subvariety $Y$ of $\mathbb{R}^{n}$ whose complement is compact. Then, for any sphere $S^{r}$, the product $S^{r} \times T$ is globally Nash isomorphic to a real algebraic variety.

Proof. Let $X$ be the algebraic Alexandrov compactification (see [BCR]) of $Y$, and let us consider $T$ canonically embedded into $X$. Since we can assume that $T$ is unbounded, $\bar{T}$ is a connected component of $X$ and $\bar{T}-T$ is a point. Thus the conclusion follows from 2.4 .

Theorem 2.6. Let $X$ be a compact connected global Nash subvariety of $\mathbb{R}^{n}$. Then, for any sphere $S^{r}$, the product $S^{r} \times X$ is globally Nash isomorphic to a real algebraic variety.

Proof. By the results of CRS there exist Nash functions $f_{1}, \ldots, f_{p}$ on $\mathbb{R}^{n}$ such that $X=\left\{x \in \mathbb{R}^{n} \mid f_{1}(x)=\cdots=f_{p}(x)=0\right\}$.

By the classical Artin-Mazur Theorem (see e.g. [BCR] there exist a nonsingular algebraic variety $N$ of $\mathbb{R}^{n+p+s}$, an open Nash embedding $\sigma: \mathbb{R}^{n} \longrightarrow N$ such that $\sigma\left(\mathbb{R}^{n}\right)$ is a connected component of $N$, and a regular map $h: N \longrightarrow \mathbb{R}^{p}$ such that $\pi \sigma=\mathrm{id}, h \sigma=f$, where $\pi$ is the canonical projection $\mathbb{R}^{n+p+s} \longrightarrow \mathbb{R}^{n}$.

Let $Z=\left\{z \in N \mid h_{1}(z)=\cdots=h_{p}(z)=0\right\}$. It follows that $\sigma(X)=\sigma\left(\mathbb{R}^{n}\right) \cap Z$ and then $\sigma(X)$ is a compact connected component of $Z$. The conclusion follows from Theorem 2.3

\section{REFERENCES}

[AK] S. Akbulut, H. King, Topology of real algebraic sets, Math. Sci. Research Institute Publ. 25, Springer, Berlin (1992) MR1225577(94m:57001)

[BCR] J. Bochnack, M. Coste, and M. F. Roy, Real algebraic geometry, Springer, Berlin, 1998 MR:1226238(94b:14055)

[CRS] M. Coste, J. Ruiz, and M. Shiota, Approximation in Nash manifolds, Amer. J. Math. 117 (1995), 905-927 MR.1342835 (96f:32015) 
[Lo] S. Łojasiewicz, Introduction to complex analytic geometry, Birkhäuser, Basel, 1991 MR.1131081 (92g:32002)

[TT] A. Tancredi and A. Tognoli, A note on global Nash subvarieties and Artin-Mazur Theorem, Boll. Unione Mat. Ital. Sez. B Artic. Ric. Mat. (8) 7-B (2004), 425-431 MR2072945 (2005e:14091)

[To1] A. Tognoli, Su una congettura di Nash, Ann. Scuola Norm. Sup. Pisa Sci. Fis. Mat. (3) 27 (1973), 167-185 MR0396571(53:434)

[To2] A. Tognoli, Algebraic approximation of manifolds and spaces, Sém. Bourbaki $\mathbf{5 4 8}$ $(1979 / 80)$

[Wa] A. H. Wallace, Algebraic approximation of manifolds, Proc. London Math. Soc. (3) 7 (1957), 196-210 MR0087205 (19:320a)

Dipartimento di Matematica e Informatica, Università di Perugia, Via Vanvitelli, 1, 06123 Perugia (PG), Italy

E-mail address: altan@unipg.it

Dipartimento di Matematica, Università di Trento, Via Sommarive, 58, 38050 Povo (TN), ITALY

E-mail address: tognoli@science.unitn.it 